\title{
Validation and Verification of Cavitation in Microchannels by using an Open Source Computational Tool
}

\author{
Gökçe Özkazanç Güç ${ }^{1}$ (iD), Levent Ünlüsoy ${ }^{2}$ (D), E. Yegan Erdem¹,3* (iD) \\ ${ }^{1}$ Mechanical Engineering Department, Bilkent University, Ankara, Turkey \\ ${ }^{2}$ Roketsan A.Ş., Ankara, TURKEY \\ ${ }^{3}$ National Nanotechnology Research Center (UNAM), Ankara, Turkey \\ *yeganerdem@bilkent.edu.tr \\ *Orcid No: 0000-0001-9852-2293
}

Received: 20 November 2020

Accepted: 26 July 2021

DOI: $10.18466 /$ cbayarfbe. 828275

\begin{abstract}
Cavitation is mostly unwanted in applications due to its unpredictable and distorting effect on fluid flow. On the other hand, its modelling is expensive in terms of time and computational power in general. Regarding this a tendency for using an open source software such as OpenFOAM is emerging as a promising tool for both predicting and analyzing cavity formation. In this study, validation and verification of an OpenFOAM solver is investigated for cavitation in microchannels. Experiments are carried out as well for comparison with computational results. During the experiments the cavity formation was efficiently captured by observing the fluorescent particle flow. Overall, computational and experimental results are compared to investigate the capability of OpenFoam for the chosen conditions.
\end{abstract}

Keywords: Cavitation, Computational Fluid Dynamics, Microchannels, Open Source,

\section{Introduction}

Cavitation, as a chaotic phenomenon and is generally defined as the phase change of the fluid flow by nucleation, growth, and collapse of a vapor bubble in regions where pressure drop is rapid and high [1]. Its damaging effects are undesired in most of the applications that include high speed fluid flow such as nozzles [2], hydraulic machinery [3] and diesel engines [4].

On the other hand, cavity formation within a microchannel can have several applications in particle and cell manipulation due to the vortex formation around them. Some studies in literature focused on forming microvortices within microfluidic channels by utilizing a cavity-like channel geometry for such applications [5-9]. For instance, Kamalakshakurup showed the controlled formation of microvortices to trap microparticles [5], Shen et al. studied the circulating particles in microvortices [6], Kim et al. utilized the formation of vortices for rapid mixing of reagents in production of hybrid nanoparticles [7], Shen et al. showed trapping of particles by using microcavities within a microchannel [8, 9]. Formation of cavity by controlling flow can also be useful for these studies which would eliminate the need for complex geometries.

Cavitating flow in microchannels has been investigated by researchers; some of this work was experimental [10, 11]; while most of them were focused on the numerical modelling of this phenomenon [12-14]. Rooze et al, studied pressure gradients in $100 \times 100 \mu \mathrm{m} 2$ channels with cavitation by using computational fluid dynamics software [12]; Egerer et al. performed large-eddy simulations for studying cavity formation in a throttle geometry where turbulence behavior is analyzed and compared with experimental work [13], and Ghorbani et al. studied cavitating nozzle flow in microchannels with high Reynolds numbers by numerical methods [14], Osterman et al. investigated the effect of channel aspect ratio on the formation of cavities by both numerically and experimentally where they used COMSOL Multiphysics software [15].

However, modeling of cavitation is expensive in terms of both computational power and time. To minimize this cost, usage of an Open-Source software is emerging [16, 18]. OpenFOAM, is becoming one of the most preferred tools due to its compatibility with $\mathrm{C}++$ language and user-friendly structure [19]. 
Different than the studies in literature, this work uses the OpenFOAM solver interPhaseChangeFOAM to examine cavity formation and verification in microchannels. Its findings can also be extended for applications in microchannels as well as in industry where a quick and low-cost analysis is required.

\section{Materials and Methods}

\subsection{Computational Model}

For solving the cavitating flow, the geometry shown in Figure 1 is used which is a modification of the former geometries studied in the literature [20-21]. Solution domain is given in Figure 1 with its dimensions. Microchannels with this geometry are fabricated for obtaining experimental results used for comparison.

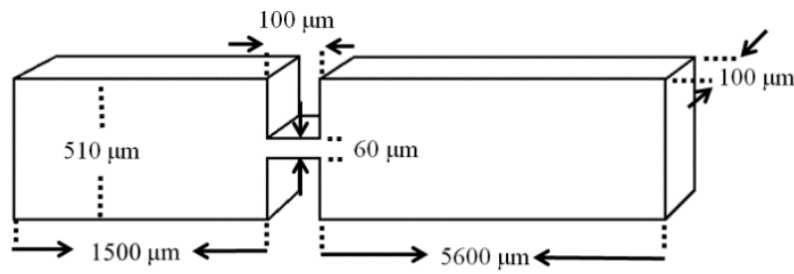

Figure 1. Geometry used for experimental and computational studies.

Mesh is generated with using OpenFOAM's blockMesh and refineMesh utilities. Since the solution of the wall is only of interest in throttle section, first layer thickness is calculated, and cells are generated regarding the minimum thickness; then refinement levels are used for solving the model. There are around $360 \mathrm{e}^{3}$ cells at the final mesh. Generated mesh is shown in Figure 2.

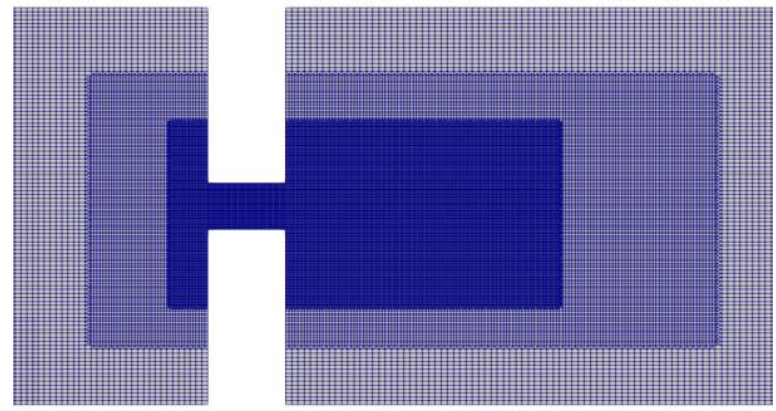

Figure 2. Mesh generated for computational solution.

Among the OpenFoam's several different solvers interPhaseChangeFoam is chosen for this case with using total pressure as inlet, static pressure as outlet and no slip as wall boundary conditions.

\subsection{Experimental Set-Up and Fabrication}

Experimental set-up used for observing the cavitation phenomena includes a pressure pump to maintain a constant fluid flow and a high-speed camera for capturing the images of flow. The schematic of the setup is shown in Figure 3.

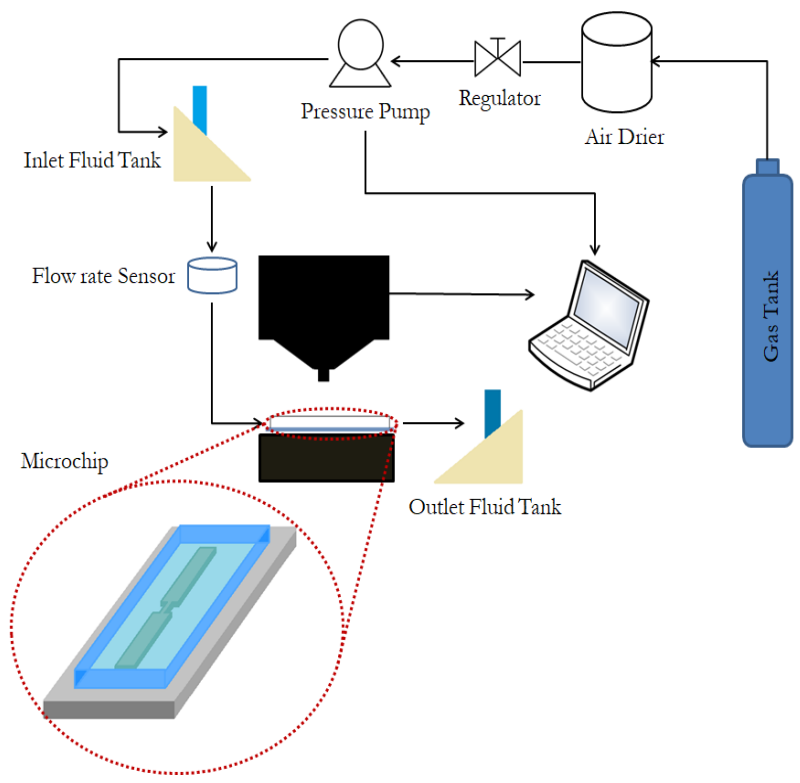

Figure 3. Schematic of the experimental set-up.

Microfluidic devices were fabricated with a conventional soft-lithography technique where initially a master mold is made in clean room by using negative photoresist SU-8 and later pouring polydimethylsiloxane (PDMS) into this mold and finally curing to obtain the channel geometry. At the last step, these channels are bonded to a glass slide for encapsulation. Figure 4 shows the fabricated PDMS channel under the optical microscope.

\section{Results and Discussion}

During the experiments, high speed camera was used to capture the fluid flow at a rate of 8-16 frames per second. Flow was maintained by supplying an input pressure at the channel entrance with a pressure pump where the value was variated from 1 bar to 6.5 bars. It is seen that for lower input pressure values, flow does not encounter any phase change, therefore cavity does not form. Representation of these flows are given in Figure 4 for 3 bars. It is seen that these flows have a regular steady state flow behavior.

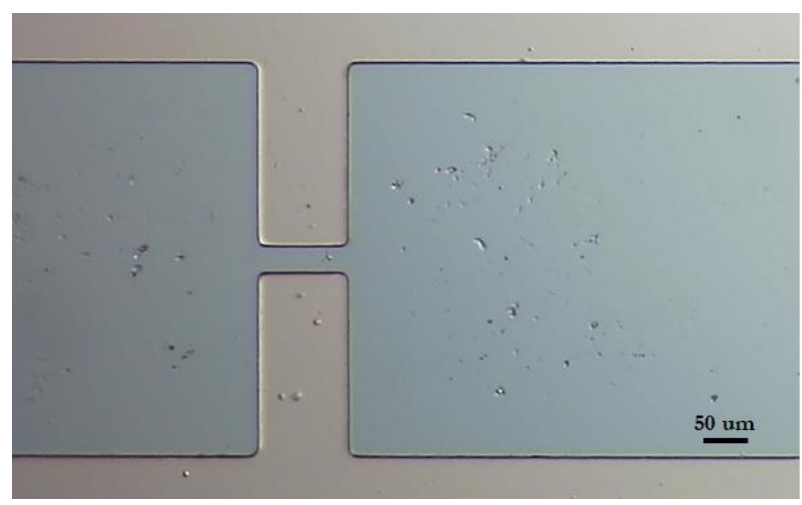

Figure 4. Steady flow with 3 bars input pressure without cavity formation. 
As the input pressure is increased however, flow characteristics change. For example, for higher pressure values (4-5 bars), bubbles begin to occur and fade away. The moment at which a bubble occurs is captured and marked in Figure 5.

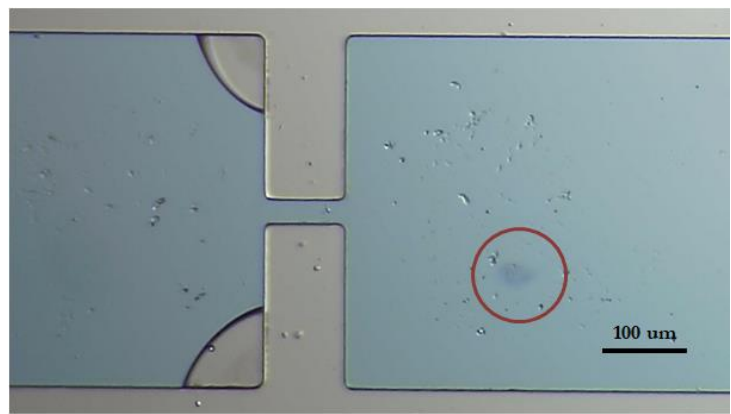

Figure 5. Vapor bubble occurrence at 4 bars.

For 5-6 bars at the inlet, number of bubbles double. Same behavior is also observed in computational results. Figure 6 a shows the experimental results while Figure $6 \mathrm{~b}$ shows the CFD results for multiple cavity formation.

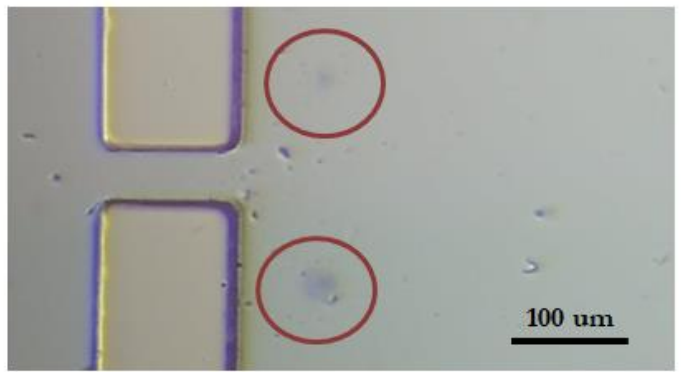

(a)

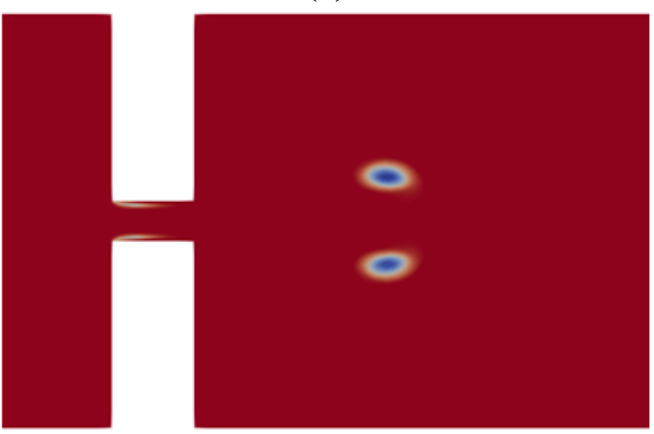

(b)

Figure 6. Comparison of simulation and experimental results. (a) Cavity formation observed during an experiment, (b) cavity formation observed in computational work.

For the higher velocities, formation of bubbles increases since pressure drops to lower values however since the flow is highly chaotic bubbles occur and disappear suddenly. Same pattern is also observed in the simulation results. An image obtained during an experiment with high velocity is given in Figure 7. From both experimental and the simulation visuals, it is seen that the flow forms a symmetrical elliptic pattern, which shows that the model is solving the flow field correctly and the given boundary conditions are satisfying the experimental conditions.

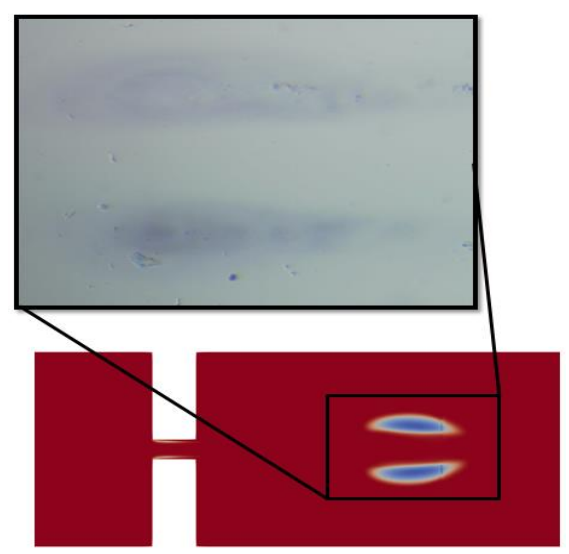

Figure 7. Low-density zones observed in experiment and simulation.

Cavitation behavior is also investigated using fluorescent latex beads, which are yellow-green carboxylate-modified polystyrene; this clarified the formation of cavity and enabled a better comparison with simulation results. With this way streamline is clearly observed unlike regular high speed camera visuals. This method has shown better performance in visualizing the experiments than any previously suggested methods in literature.

In this manner, from Figure $8 \mathrm{a}$, the region of vortex is clearly seen at the initial time steps and the movement of the vortex region is shown in Figure $8 \mathrm{~b}$. The cavity formation is observed with a darker region, where there is no liquid flow and therefore no fluorescent bead.

\section{Conclusion}

In this study, both simulations and experiments were conducted under the same boundary conditions to observe cavity formation in a microfluidic channel. The experimental set-up consisted of a pressure pump, a microfluidic chip and a high-speed camera.

The visualization of flow was facilitated by using fluorescent particles dissolved in water; which presents a novel approach for capturing the low-pressure zones in the flow.

When the experiments were compared with simulations, it is seen that there is a great resemblance between the two results. However, since cavitation is a chaotic flow, these similarities were only captured at the regions closer to the formation zone. Therefore, as a future work, by adding local pressure and velocity sensors, 
numerical data for these low-pressure regions can be obtained in addition to the image-based comparisons presented in this paper.

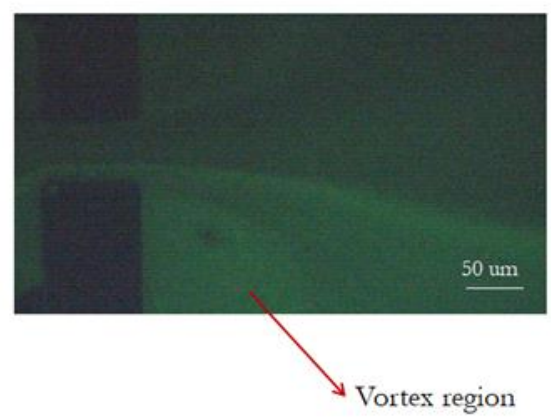

(a)
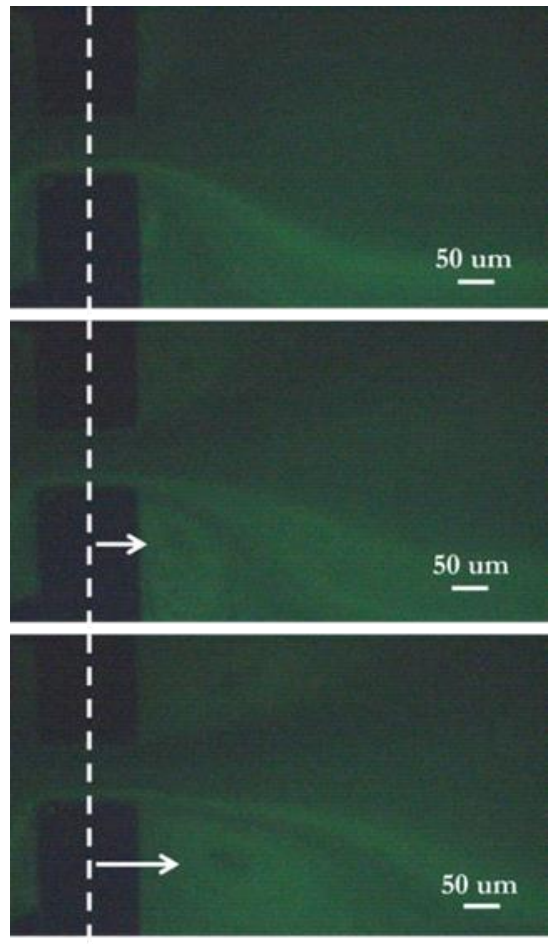

(b)

Figure 8. (a) Vortex region and (b) its movement with fluid flow.

\section{Acknowledgement}

Authors acknowledge the support from the Researcher Training Program for Defense Industry Program (SAYP) supported by the Presidency of Defense Industries in cooperation with Roketsan AŞ.

\section{Author's Contributions}

Gökçe Özkazanç Güç: Fabricated the microreactor, performed the experimental and computational work. Helped in the manuscript preparation.

Levent Ünlüsoy: Advised the research part on simulations and helped the formation of the computational model. Reviewed the final versions of the manuscript.
E. Yegan Erdem: Designed the microreactor and the experimental steps. Prepared the manuscript and the revised version.

\section{Ethics}

There are no ethical issues after the publication of this manuscript.

\section{References}

1. Brennen, C. E. Cavitation and bubble dynamics: Cambridge University Press, 2014; pp. 6.

2. Tamaki, N., Shimizu, M. Nishida, K. Hiroyasu, H. 1998. Effects of cavitation and internal flow on atomization of a liquid jet. Atomization and Sprays; 8 (2): 247-254.

3. Arndt, R. E. Cavitation in fluid machinery and hydraulic structures. 1981. Annual Review of Fluid Mechanics; 13(1): 273326.

4. Aoyama, T., Suzuki, S., Kawamoto, A., Noda, T., Ozasa, T., Kato, T., Ito, T. 2007. Preventive design and analysis of cavitation noise on diesel engine. $R \& D$ Review of Toyota $C R D L$; 40(1): 36-42.

5. Kamalakshakurup, G., Aghaamoo, M., Ataei, M., Zhang, N., Lee, A. P. 2021. Shear-dependent microvortices in liquid-liquid flowfocusing geometry: A theoretical, numerical, and experimental study. Physics of Fluids; 33: :032016.

6. Shen, F., Li, Z., Xue, S., Li, M., Liu, Z. 2021. Particle recirculating orbits within microvortices using microfluidics $J$. Phys. D: Appl. Phys.; 54: 025401.

7. Kim, Y. T., Chung, B. L., Ma, M., Mulder, W. J. M., Fayad, Z. A., Farokhzad, O. C., Langer, R. 2012. Mass production and size control of lipid-polymer hybrid nanoparticles through controlled microvortices. Nano Letters; 12(7): 3587-3591.

8. Shen, F., Xu, M., Wang, Z., Liu, Z. M. 2017. Single-particle trapping, orbiting, and rotating in a microcavity using microfluidics. Applied Physics Express; 10(9): 097301.

9. Shen, F., Xue, S., Zhou, B., Xu, M., Xiao, P., Liu, Z. 2018. Evolution of single-particle recirculating orbits within a hydrodynamic microvortex. J. Micromech. Microeng.; vol. 28(8): 085018 .

10. Ayela, F., Cherief, W., Colombet, D., Ledoux, G., Martini, M., Mossaz, S., Podbevsek, D., Qiu, X., Tillement, O. 2017. Hydrodynamic cavitation through "labs on a chip": from fundamentals to applications. Oil Gas Sci. Technol.; 72(19): 1-12.

11. Ghorbani, M., Chen, H., Villanueva, L. G., Grishenkov, D., Koşar, A. 2018. Intensifying cavitating flows in microfluidic devices with poly(vinyl alcohol) (PVA) microbubbles. Physics of Fluids, 30: 102001.

12. Rooze, J., Andre', M., van der Gulik, G.-J. S., Fernandez-Rivas, D., Gardeniers, G. E., Rebrov, E. V., Schouten, J. C., Keurentjes, J. T. F. 2012. Hydrodynamic cavitation in micro channels with channel sizes of 100 and 750 micrometers. Microfluid. Nanofluid.; 12: 499-508.

13. Egerer, C. P., Hickel, S., Schmidt, S. J., Adams, N. A. 2014 Large-eddy simulation of turbulent cavitating flow in a micro channel. Phys. of Fluids; 26(8): 085102.

14. Ghorbani, M., Yildiz, M., Gozuacik, D., Kosar, A. 2016. Cavitating nozzle flows in micro- and minichannels under the 
effect of turbulence. Journal of Mechanical Science and Technology; 30(6): 2565-2581.

15. Osterman, N., Derganc, J., Svenšek, D. 2016. Formation of vortices in long microcavities at low Reynolds number. Microfluid Nanofluid; 20(33): 1-10.

16. Cazzoli, G. Falfari, S., Bianchi, G. M., Forte, C., Catellani, C. 2016. Assessment of the cavitation models implemented in openfoam ${ }^{\circledR}$ under di-like conditions. Energy Procedia; 101: 638645.

17. Asnaghi, A., Feymark, A. Bensow, R. E. Numerical simulation of cavitating flows using openfoam, proceedings of the 18th Numerical Towing Tank Symposium, Cortona, Italy, 2015.

18. Zeng, Q., Gonzales-Avila, S. R., Ohl, C.-D. 2020. Splitting and jetting of cavitation bubbles in thin gaps. J. Fluid Mech.: 896: A28.

19. Weller, H. G., Tabor, G., Jasak, H., Fureby, C. 1998. A tensorial approach to computational continuum mechanics using objectoriented techniques. Computers in Physics; 12 (6): 620-631.

20. Medrano, M., Zermatten, P., Pellone, C., Franc, J.-P., Ayela, F. 2011. Hydrodynamic cavitation in microsystems. I. Experiments with deionized water and nanofluids. Physics of Fluids, 23(12): 127103.

21. Medrano, M., Pellone, C., Zermatten, P., Ayela, F. 2012. Hydrodynamic cavitation in microsystems. II. Simulations and optical observations. Physics of Fluids; 24(4): 047101. 